María L. Casas, Ángel Esteban, Miguel González-Muñoz, Moisés Labrador-Horrillo, Mariona Pascal* y Aina Teniente-Serra

\title{
Proyecto VALIDA: Validation of ALlergy In vitro Diagnostics Assays (Herramientas y recomendaciones para la valoración de las pruebas in vitro en el diagnóstico de la alergia)
}

https://doi.org/10.1515/almed-2020-0022

Recibido 20-2-2020; aceptado 5-5-2020; publicado

en línea 27-7-2020

Resumen: En la evaluación del paciente con sospecha de alergia las pruebas de detección y cuantificación de la inmunoglobulina $\mathrm{E}$ (IgE) específica in vitro se usan de manera habitual en los laboratorios clínicos para ayudar en el diagnóstico de la alergia. Actualmente existen diferentes alternativas comerciales para realizar estos ensayos, pero los resultados obtenidos por cada uno de ellos pueden variar, lo que condiciona el diagnóstico y el tratamiento que se le proporcionará al paciente. Con el fin de dar respuesta a los retos planteados por las diferencias entre las distintas técnicas para la determinación in vitro de la IgE específica, un grupo de expertos ha recogido en un documento una serie de recomendaciones sobre las

\footnotetext{
*Autor para correspondencia: Dra. Mariona Pascal, Sociedad Española de Alergología e Inmunología Clínica (SEAIC), Madrid, España; y Sociedad Española de Inmunología (SEI), Barcelona, España; Servei d'Immunologia, CDB, Hospital Clínic de Barcelona, IDIBAPS, Universitat de Barcelona, C/ Villarroel 170, Esc. 4, Pl. 0, 08036, Barcelona, España; and Red de Investigación ARADyAL, Instituto Carlos III, Madrid, España, E-mail: mpascal@clinic.cat, Tel.: +34 932275463/+34 690002568, Fax: +34 934518038 . María L. Casas: Sociedad Española de Medicina de Laboratorio (SEQCML), Barcelona, España; Servicio de Análisis Clínicos, Hospital Universitario Fundación Alcorcón, Alcorcón, Madrid, España Ángel Esteban: Sociedad Española de Medicina de Laboratorio (SEQCML), Barcelona, España; Servicio de Análisis Clínicos, Hospital General Universitario de Alicante, Alicante, España Miguel González-Muñoz: Sociedad Española de Inmunología (SEI), Barcelona, España; Servicio de Inmunología, Hospital Universitario La Paz, Madrid, España

Moisés Labrador-Horrillo: Sociedad Española de Alergología e Inmunología Clínica (SEAIC), Madrid, España; Servicio de Alergología, Hospital Universitari Vall d'Hebron, Barcelona, España

Aina Teniente-Serra: Sociedad Española de Inmunología (SEI), Barcelona, España; Servicio de Inmunología, LCMN, Hospital Universitari Germans Trias i Pujol, Badalona, España
}

implicaciones que puede tener el uso de una determinada técnica in vitro y el impacto en el manejo del paciente alérgico que suponen las diferencias entre las distintas técnicas. La lectura y el análisis de este documento de consenso ayudarán a entender las implicaciones que tiene el cambio de método de diagnóstico in vitro en el manejo del paciente con alergia, en su calidad de vida y en los costes socioeconómicos asociados a la enfermedad.

Palabras clave: alergia; diagnóstico in vitro; inmunoglobulina E; recomendaciones.

\section{Introducción}

A raíz del descubrimiento de la inmunoglobulina $\mathrm{E}$ (IgE) y el posterior desarrollo del primer método de diagnóstico para su determinación in vitro, ha habido un antes y un después en el diagnóstico de la patología alérgica mediada por IgE [1].

La alergia es una enfermedad que presenta una elevada prevalencia $[2,3]$, provoca un deterioro de la calidad de vida del paciente y, según el caso, puede implicar unos costes elevados, tanto directos como indirectos, para el Sistema Nacional de Salud y la sociedad $[4,5]$.

En la actualidad, en el mercado español se comercializan diferentes técnicas de determinación in vitro de $\operatorname{IgE}$ total y específica (tIgE y sIgE, respectivamente). La diversidad en la oferta de tecnologías disponibles, la complejidad de la patología alérgica y el aumento de su prevalencia [6-9] han conllevado la necesidad de establecer unas recomendaciones para evaluar de manera objetiva las metodologías disponibles en la actualidad [10].

El objetivo del presente documento es recopilar la evidencia sobre las distintas técnicas de diagnóstico in vitro actualmente disponibles, así como elaborar una serie de recomendaciones que ayuden a valorar cuál es la técnica de 
detección de IgE in vitro que mejor se ajusta a las necesidades de la práctica clínica habitual de cada centro sanitario y qué se debe tener en cuenta al plantearse un cambio.

\section{Metodología}

Para la elaboración del presente documento se conformó un grupo de trabajo integrado por especialistas que desarrollan su actividad profesional en el área de diagnóstico de la inmunoalergología. El grupo de trabajo fue designado por las 3 sociedades principales implicadas en el diagnóstico de alergia: la Sociedad Española de Medicina de Laboratorio (SEQC-ML), la Sociedad Española de Inmunología (SEI) y la Sociedad Española de Alergología e Inmunología Clínica (SEAIC). Cada sociedad eligió a dos de sus afiliados como representantes de esta, formando un panel de 6 expertos.

Se elaboró una relación de cuestiones que responder en el documento, para la que se realizaron diversas búsquedas bibliográficas. Las búsquedas se centraron en los siguientes aspectos: 1) diferencias entre las técnicas de determinación in vitro de SIgE disponibles; 2) características de la técnica de detección de sIgE in vitro ideal; 3) recomendaciones de las principales guías en las que se aborda el diagnóstico de la patología alérgica, y 4) posibles consecuencias de un cambio de técnica en el manejo del paciente a todos los niveles [4, 11-15]. Con el fin de poder cuantificar la evidencia científica se realizó una búsqueda bibliográfica de las publicaciones existentes para cada una de las diferentes técnicas de detección de sIgE in vitro comercialmente disponibles en España. La búsqueda se llevó a cabo en la base de datos Medline usando los siguientes términos en inglés para cada una de las técnicas in vitro: ("Allergy AND Immunology" [Mesh] OR Allergy) AND (ImmunoCAP NOT ISAC), (“Allergy AND Immunology" [Mesh] OR Allergy) AND (ImmunoCAP AND ISAC), (“Allergy AND Immunology" [Mesh] OR Allergy) AND (Immulite), ("Allergy AND Immunology" [Mesh] OR Allergy) AND (Euroline), ("Allergy AND Immunology" [Mesh] OR Allergy) AND (Allergy Explorer-ALEX). No se aplicaron filtros adicionales a la búsqueda. Posteriormente, se eliminaron manualmente las publicaciones que no correspondían a los criterios de búsqueda mencionados y se recogió el número de publicaciones, por técnica y por año, desde la publicación del primer trabajo de cada técnica hasta la actualidad. Por último, se buscaron los ensayos clínicos completados en los que se han utilizado las distintas técnicas de diagnóstico in vitro analizadas en la base de datos clinicaltrials.gov, utilizando los términos "nombre de la técnica”, “condición o enfermedad alérgica”, y seleccionando los estudios completados. La búsqueda fue acotada entre el 1 de enero de 1989 (año en el que se comercializó el primer reactivo para la detección de tIgE y sIgE) y el 1 de octubre de 2019 (fecha en la que se preparó este documento).

Finalmente, se debatieron los puntos clave de acuerdo con los resultados de la búsqueda bibliográfica, que fueron contrastados con la experiencia práctica de los expertos, para así consensuar las recomendaciones incluidas en el presente documento.

\section{Importancia de las técnicas in vitro en el diagnóstico de la alergia}

Según la guía de la Academia Europea de Alergia e Inmunología clínica, EAACI, [7], el protocolo de diagnóstico recomendado ante un paciente con sospecha de alergia empieza y se basa en una historia clínica completa, que dirige el posterior estudio de sensibilización, normalmente primero in vivo por prueba cutánea (skin prick test o SPT) y a continuación un estudio in vitro en suero.

El estudio alergológico in vivo centrado básicamente en las pruebas cutáneas es rápido y posee una gran sensibilidad y especificidad, pero a la vez presenta ciertas limitaciones que hacen necesario, en la mayoría de los casos, el uso combinado de técnicas in vitro. Entre las limitaciones de las pruebas cutáneas destacan, principalmente, la falta de estandarización y la variabilidad de los extractos que se utilizan, la posible subjetividad en la interpretación de los resultados (que afecta, por tanto, a la reproducibilidad), la infrecuente - aunque no descartable - posibilidad de inducir una reacción sistémica en el paciente alérgico, la imposibilidad de valorar componentes alergénicos recombinantes, y la necesidad de retrasar su implementación en pacientes con diversas afecciones cutáneas (dermografismo, dermatitis, urticaria) y en tratamiento con antihistamínicos u otros fármacos [16].

Debido a todos los aspectos mencionados anteriormente, el estudio in vitro ha ido adquiriendo una importancia creciente en la última década, al ampliar las posibilidades y la especificidad diagnóstica en el campo de la alergología, y disminuir los riesgos para el paciente derivados de una posible reactividad cruzada o una clínica no concordante con los datos de sensibilización obtenidos en el laboratorio [17].

Las aportaciones del diagnóstico molecular de la alergia han sido ampliamente documentadas durante las últimas décadas. En este sentido, el documento de 
consenso publicado por la WAO-ARIA-GA²LEN [10], sostiene que el diagnóstico in vitro desempeña un papel importante en 3 fases clave del diagnóstico de la alergia: 1) distinción entre sensibilización genuina y reactividad cruzada; 2) evaluación del riesgo de nuevas reacciones sistémicas en casos seleccionados de alergia a alimentos, y 3) identificación de los mejores pacientes candidatos a recibir inmunoterapia. Por este motivo, es necesario conocer las diferencias existentes entre las distintas técnicas in vitro respecto al diagnóstico.

Para entender mejor los conceptos más relevantes sobre las pruebas diagnósticas basadas en la detección de sIgE in vitro, es importante conocer los términos más habituales en el manejo de estas tecnologías [18]:

\section{Determinación de IgE in vitro}

Cuantificación de la concentración de IgE sérica en una muestra de sangre de un individuo. Puede determinarse la tIgE, es decir, toda la carga de IgE del paciente independientemente de su especificidad, o sólo la sIgE frente a un determinado extracto o componente alergénico.

\section{Alérgeno}

Es el antígeno capaz de inducir una respuesta inmunológica que conlleva la producción de anticuerpos de tipo IgE en un organismo predispuesto después del primer contacto, y posteriormente ocasionar una reacción antígenoanticuerpo que puede desencadenar síntomas clínicos si existe una nueva exposición (reacción de hipersensibilidad tipo I, o alérgica). Son los antígenos de la respuesta alérgica.

\section{Extracto total}

Un extracto alergénico total es una solución acuosa, glicerinada o un liofilizado de proteínas que proviene de la extracción (habitualmente acuosa) de una fuente alergénica completa (p. ej., cacahuete o polen de olivo).

\section{Componentes de alérgeno}

Son los componentes individuales de una fuente alergénica que reaccionan con la sIgE. La mayoría son proteínas con capacidad tanto de inducir como de desencadenar una reacción alérgica. Así pues, una fuente alergénica (p. ej., un alimento o un polen) debe considerarse como una mezcla de diferentes "componentes" alergénicos.

\section{Diagnóstico resuelto por componentes (CRD, según sus siglas en inglés)}

El diagnostico resuelto por componentes, también llamado diagnóstico molecular, detecta y cuantifica los niveles de anticuerpos SIgE para un determinado componente individual, lo que proporciona no sólo un diagnóstico mucho más preciso del paciente alérgico, sino también la posibilidad de evaluar el riesgo de nuevas reacciones e identificar los mejores candidatos para propósitos terapéuticos.

\section{Diferencias entre las técnicas de diagnóstico in vitro de la alergia}

Las técnicas de detección in vitro de la sIgE se basan en la unión de un determinado alérgeno a una fase sólida o líquida, a la que se unirá la sIgE del paciente para dicho alérgeno, tras la incubación con el suero de éste. Las moléculas de IgE no específicas para el alérgeno en cuestión se eliminarán mediante un lavado. Posteriormente se incubará el complejo sIgE-alérgeno con un anticuerpo antiIgE marcado que permitirá detectarlos. La señal emitida por el anticuerpo marcado permitirá medir la concentración de sIgE [19]. Los inmunoensayos para SIgE requieren una curva estándar de calibración para determinar la cantidad de IgE presente en el suero del paciente realizada a partir de unos calibradores concretos. Dichos calibradores para la IgE total están estandarizados respecto a la Preparación de Referencia Internacional para IgE Humana de la Organización Mundial de la Salud [20]. Esta se usa para interpolar resultados a $\mathrm{kU}_{\mathrm{A}} / \mathrm{L}$ de $\mathrm{sIgE}$, donde una unidad equivale a 2,4 ng de IgE. Existe evidencia de que una unidad de $\mathrm{kU}_{\mathrm{A}} / \mathrm{L}$ de sIgE equivale a una unidad de $\mathrm{kU} / \mathrm{L}$ de tIgE [21].

En los últimos años, los avances en la tecnología y la creciente importancia del diagnóstico in vitro de la alergia han dado lugar al desarrollo de varias técnicas, basadas en el sistema de detección de IgE descrito en el párrafo anterior, ya disponibles en el mercado español: ImmunoCAP ${ }^{\mathrm{TM}}$ e ImmunoCAPTM ISAC (Thermo Fisher Scientific), Immulite $^{\circledast}$ (Siemens), Euroline ${ }^{\circledast}$ (Euroimmun) y ALEX $^{\circledast} /$ ALEX $^{2 \circledast}$ (Macro Array Diagnostics).

El análisis de las características de las distintas técnicas de diagnóstico in vitro de la alergia muestra que, pese a que todos los ensayos están basados en un reconocimiento antígeno-anticuerpo, éstos difieren en los métodos de unión a alérgenos, los métodos de detección de la señal, el volumen de muestra necesario, el tipo de cuantificación y el grado de automatización [22-25]. El estudio alergológico 
in vitro se puede realizar mediante técnicas singleplex, que permiten la detección de niveles de sIgE frente a un único alérgeno en concreto o fuente alergénica, o multiplex, que consiguen medir la presencia de sIgE frente a una batería de alérgenos de manera simultánea [26].

A continuación, se especifican algunas características de cada una de ellas:

\section{Sistemas singleplex}

El método ImmunoCAP ${ }^{\mathrm{TM}}$ presenta un catálogo de más de 600 alérgenos (entre los que se incluyen más de 100 componentes moleculares) para los que realiza una determinación cuantitativa (fluoroenzimoinmunoensayo).

Immulite $^{\circledast}$ es un método que dispone de más de 480 extractos alergénicos diferentes y 33 componentes moleculares. La tecnología utilizada es un enzimoinmunoensayo por quimioluminiscencia, con resultados cuantitativos.

\section{Sistemas multiplex}

El ensayo multiplex ImmunoCAP ${ }^{\mathrm{TM}}$ ISAC utiliza una matriz fija de 112 componentes alergénicos recombinantes o nativos purificados fijados por triplicados, cuya determinación es semicuantitativa (fluorescencia).

El método ALEX ${ }^{\circledast} /$ ALEX $^{2 \circledast}$ utiliza una matriz fija de más de 120 extractos alergénicos y 170 componentes moleculares; se obtienen resultados semicuantitativos para IgE total y cuantitativos para sIgE, mediante la tecnología de inmunoensayo en fase sólida (colorimetría).

Euroline $^{\circledast}$ es una técnica que ofrece la determinación de alrededor de 100 paneles distintos de alérgenos (que incluyen extractos totales y componentes), con resultados semicuantitativos mediante la tecnología de inmunoensayo en fase sólida (colorimetría).

En la Tabla 1 se incluye un resumen de las principales características de cada una de las técnicas.

Una prueba diagnóstica debe ofrecer un balance óptimo desde el punto de vista de sensibilidad y especificidad, y disponer de evidencia científica que avale su uso. Además, sería conveniente que cubriera un amplio abanico de alérgenos y que permitiera la automatización. Crameri define el ImmunoCAP como la técnica gold standard de diagnóstico in vitro [27], a la espera de nueva evidencia científica respecto al resto de técnicas. Por otro lado, es la técnica de referencia con la que se comparan las demás en los estudios de correlación [28-30].

\section{Consideraciones ante un cambio de técnica in vitro}

\section{Correlación entre las diferentes técnicas}

La evidencia científica ha permitido comprobar que los resultados de las diferentes técnicas in vitro no son comparables ni intercambiables [23, 31-33]. Aunque en algunos casos podamos encontrar cierta correlación entre técnicas, los resultados no son intercambiables mediante el uso de factores de conversión, ya que, como se ha comentado anteriormente, no disponemos de unidades referidas a un estándar común [23, 33-37]. A modo de ejemplo, el estudio comparativo entre Immulite e ImmunoCAP llevado a cabo por Wood y col. [35] puso de manifiesto que no hay ningún método validado en el diagnóstico in vitro de la alergia que correlacione los resultados de las diferentes técnicas de forma fiable; asimismo, los datos obtenidos mediante una técnica no se pueden reproducir en otra técnica. Las repercusiones clínicas de la cuantificación de sIgE son importantes, por lo que el uso de uno u otro método es una decisión relevante.

\section{Fuentes de alérgenos: calidad y reproducibilidad}

La calidad del alérgeno utilizado en cada técnica varía debido a varios factores, entre ellos, la temporada en la que se recoge la materia prima (p. ej., pólenes), el sistema de conservación del material, el grado de dificultad de su identificación, la contaminación con otras fuentes alergénicas que pueden dar lugar a una reactividad cruzada y las diferencias en la metodología de extracción (producción recombinante frente a purificación) entre los diferentes fabricantes [38]. Por todo ello, es necesario que los alérgenos sean sometidos a un exhaustivo y riguroso control de calidad.

También es importante conocer si el alérgeno contiene cross-reactive carbohydrate determinats (CCD) que son oligosacáridos presentes en muchos alérgenos con alta reactividad cruzada y escasa relevancia clínica, pudiendo dar lugar a falsos positivos. Con respecto a la prevalencia de estos antígenos y su impacto, se estima que estos pueden llegar a presentar reactividad en un $7,5-35 \%$ los pacientes, por lo que puede representar un problema a la hora del diagnóstico [39-41]. En la actualidad se aplican tres estrategias para evitar falsas positividades por CCDs: 1 ) producción recombinante de los alérgenos (en el caso de los componentes), 2) uso de inhibidores de CCDs durante el 
proceso de detección de sIgE o 3) uso de MUXF3 (alérgeno compuesto exclusivamente por epítopos carbohidrato presente en muchas glicoproteínas vegetales) como control de positividad por CCDs. Con el uso de estas estrategias se observa una disminución de los falsos positivos y, por lo tanto, un aumento de la precisión diagnóstica [41-43].

\section{Diversidad de catálogo}

Es importante que el catálogo de alérgenos presente variedad tanto de extractos totales como de componentes moleculares, puesto que ambos son complementarios para un correcto diagnóstico. Es decir, saber a qué fuente alergénica estamos sensibilizados, y específicamente a cuál o cuáles de los distintos componentes alergénicos de esta fuente, es una información de gran valor clínico [44, 45].

En el día a día de la práctica clínica, disponer de un catálogo amplio puede contribuir a diagnosticar a más pacientes, ya que estamos ante una patología variable que puede resultar muy compleja en algunos casos [46]. Esto es especialmente relevante en nuestro entorno, ya que en España la polisensibilización representa un problema grave, igual que en otros países del entorno mediterráneo, como Italia y Grecia [47].

\section{Diferencias en la literatura científica}

Valorar la evidencia científica disponible respecto a una metodología permite al profesional de la salud tomar decisiones más objetivas. Por eso es importante conocer las diferencias entre las distintas técnicas disponibles.

Antes de seleccionar un método para el diagnóstico in vitro de la alergia, es necesario asegurar que éste disponga de una evidencia científica suficiente proveniente de estudios, cuyo fin sea la mejora en el manejo del paciente alérgico. Lo contrario puede llevar a un retraso y una menor precisión en el diagnóstico, lo que podría repercutir en la calidad de vida del paciente.

Es por este motivo que se realizó una búsqueda de la literatura científica publicada sobre las distintas técnicas de detección de sIgE in vitro. Los resultados se detallan a continuación:

Siguiendo el orden cronológico de aparición de la evidencia de cada técnica de diagnóstico in vitro de alergia, y utilizando los criterios de búsqueda descritos anteriormente (ver apartado de metodología), se observa que en el momento en que se redactó este documento ImmunoCAP (1990) presenta más de 600 publicaciones en la base de datos Medline, Immulite (1996) ha sido 
referenciado en 52 artículos indexados, ImmunoCAP ISAC (2010) aparece en 117 artículos, Euroline (2018) en 7 artículos, y ALEX (2018) es la técnica utilizada en 3 publicaciones (Tabla 2, Figura 1). Debido al impacto de la localización geográfica en los perfiles de sensibilización, más allá de valorar el número de publicaciones, también es muy importante disponer de evidencia local: ImmunoCAP e ImmunoCAP ISAC son las técnicas que disponen de una mayor evidencia hasta el momento (Material Suplementario, Tabla 1). ImmunoCAP e ImmunoCAP ISAC disponen de más de 45 y 12 estudios completados en España, respectivamente. Immulite se ha utilizado en 2 estudios nacionales (Material Suplementario, Tablas 1 y 2).

\section{Impacto del cambio de método de diagnóstico in vitro}

En la valoración del impacto del cambio de método de determinación de sIgE in vitro siempre hay que considerar que una prueba positiva sólo indica sensibilización, y sólo si está asociada a reactividad clínica (síntomas) podemos hablar de alergia [48]. No existen puntos de corte universales para los diferentes alérgenos que permitan predecir la probabilidad de reactividad clínica asociada a un determinado valor de sIgE, por lo que éstos se deben validar clínicamente para cada técnica. Si un laboratorio cambia de técnica, sería necesario volver a establecer los valores de referencia, ya que los anteriores no serán válidos. En este sentido, es importante que haya comunicación con el clínico para establecer cómo se va a hacer la transición y establecer nuevos valores de referencia. Por tanto, tras el cambio de una técnica por otra, será necesario revisar cómo los resultados obtenidos se aplican en la práctica clínica diaria [23, 29, 32].

\section{Impacto en el diagnóstico inicial del paciente}

Las diferencias entre métodos diagnósticos pueden afectar a la interpretación de los resultados y causar confusión con respecto a los protocolos establecidos en los laboratorios y servicios clínicos que manejan al paciente alérgico. Por ejemplo, los valores de corte utilizados para las pruebas de provocación oral propuestos a partir de la experiencia acorde a los resultados de una técnica de diagnóstico in vitro determinada, no son válidos para las distintas técnicas para su uso en la toma de decisiones clínicas. Así, las pruebas de provocación con alimentos se basan en puntos de corte locales; cada centro utiliza puntos de corte propios que, en caso de que cambie la metodología, deberían establecerse de nuevo [23]. Esto tiene diversas implicaciones: aumento de los costes asociados al diagnóstico, molestias para el paciente, riesgos para la seguridad del paciente y sobrecarga del sistema de salud.

\section{Impacto en la toma de decisiones terapéuticas}

Hoy en día, se considera que el único tratamiento capaz de modificar el curso natural de la enfermedad alérgica es la desensibilización o la inmunoterapia específica con alérgeno (ITA). En el caso de la ITA, se ha demostrado que puede prevenir la progresión de la enfermedad, y su efecto terapéutico perdura incluso tras haber finalizado su administración [49]. Como su nombre indica, la ITA es específica para un extracto alergénico determinado, por lo que la identificación del alérgeno causante de la enfermedad es esencial para la prescripción más adecuada del tratamiento.

Por este motivo, la determinación de sIgE frente a extracto total, complementado con el diagnóstico in vitro, puede mejorar la selección del paciente para la indicación de ITA; por ejemplo, en la alergia a pólenes [50, 51] y al veneno de himenópteros [52]. Por tanto, es aconsejable disponer de un único método que permita la realización en paralelo de componentes y extractos totales, para poder diferenciar a los pacientes alérgicos con múltiples sensibilizaciones genuinas de aquellos con multisensibilización por reactividad cruzada [53], sobre todo en los países con alta prevalencia de pacientes polisensibilizados [54].

Tabla 2: Comparativa de las diferentes técnicas in vitro en función de la evidencia científica disponible.

\begin{tabular}{lrrr}
\hline Técnica & $\begin{array}{r}\text { No total de publi- } \\
\text { caciones en } \\
\text { Medline }\end{array}$ & $\begin{array}{r}\text { Tasa de } \\
\text { publicación }^{\text {a }}\end{array}$ & $\begin{array}{r}\text { Estudios clínicos } \\
\text { (total de } \\
\text { pacientes } \\
\text { incluidos) }\end{array}$ \\
\hline $\begin{array}{l}\text { ImmunoCAP } \\
\text { Immulite }\end{array}$ & 633 & 22,1 & $22(2.381)$ \\
2000 & 52 & 2,16 & $1(102)$ \\
$\begin{array}{l}\text { ImmunoCAP } \\
\text { ISAC }\end{array}$ & 117 & 13 & 0 \\
$\begin{array}{l}\text { Euroline } \\
\text { Allergy }\end{array}$ \\
$\begin{array}{l}\text { Explorer- } \\
\text { ALEX }\end{array}$
\end{tabular}

El número de estudios clínicos corresponde a los que se aplican a la enfermedad alérgica y que ya están completados y publicados en

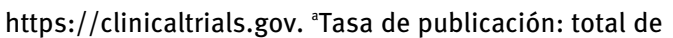
publicaciones/años desde la primera publicación. 


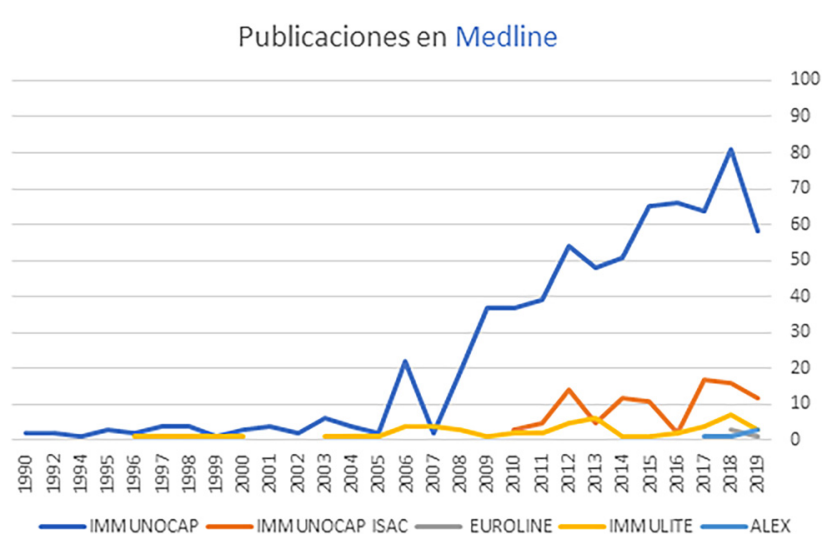

Figura 1: Gráfico de publicaciones en Medline por técnica de diagnóstico in vitro y por año.

Además, el diagnóstico in vitro puede permitir evaluar el riesgo de reacciones adversas a la inmunoterapia con algunas fuentes alergénicas, como el polen de olivo en zonas de alta exposición polínica, ya que la sensibilización a determinados componentes alergénicos (p. ej., a Ole e 9 y Ole e 7), está asociada a este tipo de reacciones $[55,56]$.

\section{Impacto en el seguimiento}

El cambio de técnica de diagnóstico in vitro durante el seguimiento de un paciente alérgico y pendiente de revisión puede suponer un problema más importante que el que representaría para el paciente diagnosticado por primera vez. Esta afirmación coincide con la literatura disponible, en la que se aconseja que, debido a la variabilidad entre Immulite y otros ensayos de sIgE, es preferible usar un solo ensayo para controlar la evolución de la alergia [28, 34, 37, 51].

Otros ejemplos los podemos encontrar en los casos en el que el seguimiento del paciente implica la realización de prueba de tolerancia a alimentos tras un periodo de evitación, según unos determinados puntos de corte de sIgE, después del diagnóstico de alergia alimentaria [57].

\section{Impacto en el coste asociado de la enfermedad alérgica}

En la alergia, como en cualquier patología, tanto a la hora de elegir un método diagnóstico como un tratamiento, se evalúa fundamentalmente su relación coste-efectividad. Esto conlleva que, en el proceso de adquisición de un método, los criterios que se tienen en cuenta son tanto técnicos como económicos (estos últimos normalmente limitados al precio unitario de la prueba). Esta aproximación, dentro del marco de la contratación pública, permite a los profesionales de la salud valorar cuál de los métodos disponibles aporta la mejor relación coste-efectividad.

Es importante que los especialistas que trabajan en el área de la inmunoalergología conozcan en profundidad las características técnicas de cada una de las metodologías disponibles, así como las diferencias entre ellas, lo que permitirá entender las implicaciones de un cambio de técnica y los costes derivados de ello, tanto directos como indirectos [58, 59].

Hasta la fecha, los estudios de coste-efectividad publicados se centran en el rendimiento de ImmunoCAP ISAC y SPT. En 2016 el NICE analizó en una revisión sistemática 4 estudios que las incluían [60]. En diversos trabajos de Hermanasen y col $(2012,2013)$ se compara el coste efectividad del ImmunoCAP ISAC frente provocación oral controlada con doble ciego y el SPT en niños con alergia a los cacahuetes. Para ello se usó un modelo de Markov a 5 años, siendo el ImmunoCAP ISAC el más eficiente frente a la provocación y el SPT [61, 62]. Glaumann y colaboradores (2013) también estudiaron el coste efectividad del ImmunoCAP ISAC frente a la provocación oral y el SPT, mostrando de nuevo ImmunoCAP dominancia sobre el resto de técnicas [63]. Por otra parte Hermansson y col. (2012) [64] y Mascialino y col. (2013) [65] analizaron el coste efectividad de ImmunoCAP ISAC frente a SPT en una población alérgica al polen, usando un modelo de Markov con un horizonte a 9 años y observaron que la suma de ImmunoCAP ISAC y SPT reduce la prescripción de inmunoterapia frente a la STP sola.

\section{Discusión}

Hasta la fecha, la legislación europea de productos sanitarios y a diferencia de la Food and Drug Administration, no exige a los productos sanitarios una validación mediante ensayos clínicos realizados en pacientes previa a la comercialización [66], diferenciándose de esta manera de los fármacos. El único requisito para su comercialización en Europa es la obtención del marcado CE. Para esto se requiere al fabricante presentar un estudio de comparación con otro método y demostrar que es reproducible en el tiempo. Dicha comparación debe aparecer en las instrucciones de uso del producto. En este sentido, es importante tener en cuenta que el estudio in vitro de detección de sIgE presenta particularidades relacionadas con la naturaleza de la IgE respecto al estudio de otros parámetros analíticos. Por este motivo, resulta importante 
conocer bien las técnicas de diagnóstico in vitro de detección de sIgE a la hora de decidir la mejor opción.

Por otra parte, se ha descrito que un diagnóstico impreciso tiene consecuencias en distintos ámbitos, con su correspondiente impacto económico:

- Laboratorio. Hay que considerar el coste y el tiempo necesarios para realizar una validación clínica exhaustiva de una nueva técnica de diagnóstico in vitro. Como ya se ha explicado anteriormente, la validación necesaria para la comercialización de un producto de diagnóstico in vitro no requiere la realización de ensayos clínicos con pacientes. Esta situación normativa implica que es responsabilidad del propio laboratorio $\mathrm{y}$, por extensión, de todos los implicados en el diagnóstico del paciente alérgico, diseñar y ejecutar una validación clínica que tenga en cuenta la realidad poblacional para verificar que el nuevo método de diagnóstico para detección de IgE cumple con los requisitos necesarios, tanto de panel de alérgenos como de sensibilidad y especificidad analíticas.

- Clínico. Un cambio de método diagnóstico puede dar pie a la obtención de resultados no concluyentes, si no se establecen los nuevos valores de referencia, lo que hace necesario el uso de más recursos para contrastar los resultados. Esto conllevaría un aumento en el gasto, tanto en número de determinaciones como en dedicación por parte del clínico.

- Logístico. Si la nueva técnica seleccionada no incluye en su portafolio los alérgenos necesarios y previamente utilizados, será necesario en muchos casos externalizar la determinación de sIgE, con el correspondiente coste asociado y la posible demora de los resultados en el tiempo.

Aunque este trabajo se ha centrado en los costes directos, también sería necesario valorar los posibles efectos en la calidad de vida de los pacientes, así como los costes indirectos que, aunque no se han descrito en este apartado, también pueden ser considerables [56].

Por otra parte, cabe destacar que la falta de estudios de comparación head to head entre las distintas técnicas ha sido una limitación a la hora de elaborar este manuscrito. Además, no tan solo faltan comparativas head to head, sino también literatura en general para muchas de las técnicas comercializadas más recientemente. Es por este motivo que se dispone de escasas herramientas para valorar un cambio de técnica y por ende un manuscrito de estas características era necesario. Otras limitaciones en el campo de la alergología, que dificultan la valoración de la prueba de detección de
sIgE in vitro más adecuado para cada laboratorio, son la variabilidad de los paneles de alérgenos en el caso del diagnóstico molecular, la falta de estandarización de los alérgenos y por tanto su reproducibilidad y la falta de controles de calidad. [67, 68].

\section{Conclusiones}

- En la actualidad existen distintas técnicas para la determinación de sIgE in vitro, y es importante conocer sus características para establecer un correcto diagnóstico y manejo del paciente alérgico.

- Tanto las técnicas singleplex como las multiplex son útiles en el diagnóstico alergológico, y su uso dependerá de la complejidad de cada caso y de las necesidades específicas del paciente.

- La evidencia científica disponible y la experiencia acumulada en la práctica clínica habitual permiten afirmar que los resultados de los diferentes métodos comercializados para el diagnóstico alergológico in vitro no son intercambiables; por tanto, es importante mantener el mismo método de detección de sIgE durante el diagnóstico, el tratamiento y el seguimiento del paciente.

- Cualquier cambio de método diagnóstico in vitro implica ineludiblemente realizar una validación exhaustiva por parte del laboratorio y los especialistas que controlan la patología alérgica del paciente.

- Un cambio de técnica puede tener implicaciones en el manejo del paciente alérgico que impacten negativamente en distintos niveles, además de suponer un incremento de costes asociados, tanto directos como indirectos.

- Una técnica de calidad es la que presenta un balance correcto entre sensibilidad y especificidad analítica, dispone de evidencia científica suficiente que avale su utilidad clínica, tiene un amplio catálogo de alérgenos y es coste-efectiva.

En la actualidad, a falta de más evidencia respecto a las tecnologías más novedosas, ImmunoCAP es la técnica que se ajusta mejor a los aspectos valorados en este documento, y que aporta mayor aval científico para el diagnóstico de la alergia in vitro en la población general.

Agradecimientos: Los autores quieren agradecer a Thermo Fisher el apoyo recibido en la realización de este manuscrito, así como a Ediciones Mayo en la asistencia editorial del mismo. 
Financiación de la investigación: Los autores declaran haber recibido honorarios por consultoría y soporte para asistencia a reuniones de trabajo relacionadas con este documento por parte de Thermo Fisher Diagnostics S.L.U. Contribución de los autores: Todos los autores han aceptado la responsabilidad del contenido completo del manuscrito y aprueban su envío.

Conflicto de intereses: Los autores declaran no tener ningún conflicto de intereses.

\section{Referencias}

1. Johansson SGO. The discovery of IgE. J Allergy Clin Immunol 2016; 137:1671-3.

2. Jenmalm MC. The mother-offspring dyad: microbial transmission, immune interactions and allergy development. J Intern Med 2017;282:484-95.

3. Gaig P, Lleonart R, Muñoz-Lejarazu D, Olona M. Prevalencia de alergia en la población adulta Española. alergol(allergy prevalence in the Spanish adult population. allergy). e Inmunol Clin 2004;19:209.

4. EAACI (European Academy of Allergy and Clinical Immunology). Immunology c. food allergy and anaphylaxis guidelines translating knowledge into clinical practice. EAACI Guidel 2014; 188. https://doi.org/10.1111/all.12437.

5. Bosnic-Anticevich S, Costa E, Menditto E, Lourenço O, Novellino E, Bialek S, et al. ARIA pharmacy 2018 "Allergic rhinitis care pathways for community pharmacy") POLLAR (impact of air pollution on asthma and rhinitis) GARD demon. Allergy Eur J Allergy Clin Immunol 2019;74:1219-36.

6. Sicherer SH, Sampson HA. Food allergy: a review and update on epidemiology, pathogenesis, diagnosis, prevention, and management. J Allergy Clin Immunol 2018;141:41-58.

7. Wang ML, Song QL, Wu HR, Ding BF, Gao XD, Sun XY, et al. Smallmolecular organic solar cells with C60/Al composite anode. Org Electron 2007;8:445-9.

8. Pridgeon C, Wild G, Ashworth F, Egner W, Ward AM. Assessment of latex allergy in a healthcare population: are the available tests valid? Clin Exp Allergy 2000;30:1444-9.

9. Lieberman JA, Sicherer SH. Diagnosis of food allergy: epicutaneous skin tests, in vitro tests, and oral food challenge. Curr Allergy Asthma Rep 2011;11:58-64.

10. Canonica GW, Ansotegui IJ, Pawankar R, Schmid-Grendelmeier $P$, Van Hage M, Baena-Cagnani CE, et al. A WAO-ARIA-GA2LEN consensus document on molecular-based allergy diagnostics. World Allergy Organ J 2013;6:1.

11. Hellings PW, Seys SF, Marien G, Agache I, Canonica W, Gevaert P, et al. ARIA masterclass 2018: from guidelines to real-life implementation. Rhinology.2019;57:392-9.

12. Scadding GK, Kariyawasam HH, Scadding G, Mirakian R, Buckley RJ, Dixon T, et al. BSACl guideline for the diagnosis and management of allergic and non-allergic rhinitis (Revised Edition 2017; 1st ed. 2007). Clin Exp Allergy 2017;47:856-89.

13. NIAID-Sponsored Expert Panel, Boyce JAA, Assaad A, Burks AW, Jones SMM, Sampson HAA, et al. Guidelines for the diagnosis and management of food allergy in the United States: report of the
NIAID-sponsored expert panel [Internet]. Elsevier Ltd. 2010. https://dx.doi.org/10.1016/j.jaci.2010.10.007.

14. Custovic A, Johnston SL, Pavord I, Gaga M, Fabbri L, Bel EH, et al. EAACI position statement on asthma exacerbations and severe asthma. Allergy Eur J Allergy Clin Immunol 2013;68:1520-31.

15. Brigham EP, West NE. Diagnosis of asthma: diagnostic testing. Int Forum Allergy Rhinol 2015;5:S27-30.

16. Jensen-Jarolim E, Jensen AN, Canonica GW. Debates in allergy medicine: molecular allergy diagnosis with ISAC will replace screenings by skin prick test in the future. World Allergy Organ J 2017;10. https://doi.org/10.1186/s40413-017-0162-3.

17. Jakob T, Forstenlechner P, Matricardi P, Kleine-Tebbe J. Molecular allergy diagnostics using multiplex assays: methodological and practical considerations for use in research and clinical routine. Allergo J Int 2015;24:320-32.

18. Plaza AM, Martin MA. Tratado de alergología pediátrica, $3^{\mathrm{a}}$ ed. Barcelona: Editorial Ergon; 2019.

19. Hamilton RG, Franklin Adkinson N. In vitro assays for the diagnosis of IgE-mediated disorders. J Allergy Clin Immunol 2004;114:213-25.

20. Hamilton RG, Oppenheimer J. Serological IgE analyses in the diagnostic algorithm for allergic disease. J Allergy Clin Immunol Pract [Internet] 2015 [cited 2019 Dec 18];3:833-40.

21. Kober A, Perborn H. Quantitation of mouse-human chimeric allergen specific IgE antibodies with immunoCAPTM technology. J Allergy Clin Immunol 2006;117:S219.

22. Hamilton RG, Williams PB. Human IgE antibody serology: a primer for the practicing North American allergist/immunologist. J Allergy Clin Immunol 2010;126:33-8.

23. Goikoetxea MJ, Sanz ML, García BE, Mayorga C, Longo N, Gamboa PM. Recommendations for the use of in vitro methods to detect specific immunoglobulin E: are they comparable? J Investig Allergol Clin Immunol 2013;23:448-54.

24. Li TM, Chuang T, Tse S, Hovanec-Burns D, El Shami AS. Development and validation of a third generation allergenspecific IgE assay on the continuous random access IMMULITE ${ }^{\circledast}$ 2000 analyzer. Ann Clin Lab Sci 2004;34:67-74.

25. Cox L, Williams B, Sicherer S, Oppenheimer J, Sher L, Hamilton R, et al. Pearls and pitfalls of allergy diagnostic testing: report from the American college of allergy, asthma and immunology/ American academy of allergy, asthma and immunology specific IgE test task force. ann. allergy. Asthma Immunol 2008;101:580-92.

26. Lee JH, Park HJ, Park KH, Jeong KY, Park JW. Performance of the PROTIA $^{\mathrm{TM}}$ allergy- $\mathrm{Q}^{\circledR}$ system in the detection of allergen-specific IgE: a comparison with the mmunoCAP ${ }^{\circledR}$ system. Allergy Asthma Immunol Res 2015;7:565-72.

27. Crameri $R$. The crux with a reliable in vitro and in vivo diagnosis of allergy. Allergy Eur J Allergy Clin Immunol 2013; 68:693-4.

28. Park KH, Lee J, Lee SC, Son YW, Sim DW, Lee JH, et al. Comparison of the immunoCAP assay and advansure ${ }^{\mathrm{TM}}$ alloscreen advanced multiplex specific IgE detection assay. Yonsei Med J 2017;58: 786-92.

29. Park KH, Lee J, Sim DW, Lee SC. Comparison of singleplex specific ige detection immunoassays: immunoCAP phadia 250 and immulite 2000 3allergy. Ann Lab Med [Internet] 2018;38:23-31. Available from: https://www.ncbi.nlm.nih.gov/pubmed/ 29071815. 
30. Dhillon RS, Fairley JW, Dhillon RS, Fairley JW. Clinical features of allergic rhinitis. Mult Quest Otolaryngol 1989;124. https://doi. org/10.4168/aard.2015.3.2.116.

31. Bojcukova J, Vlas T, Forstenlechner P, Panzner P. Comparison of two multiplex arrays in the diagnostics of allergy. Clin Transl Allergy 2019;9:1-6.

32. Sato K. Evaluation of two serum specific IgE measurement kits for patients with an allergic disease measuring equipment and reagent (Tables 1 and 2). [Igakukensa (Japanese Journal of Medical Technology)] 2010;59.

33. Yang J, Lee H, Choi AR, Park KH, Ryu JH, Oh EJ. Comparison of allergen-specific IgE levels between immulite 2000 and imunoCAP systems against six inhalant allergens and ten food allergens. Scand J Clin Lab Invest 2018;78:606-12.

34. Wang J, Godbold JH, Sampson HA. Correlation of serum allergy (IgE) tests performed by different assay systems. J Allergy Clin Immunol 2008;121:1219-24.

35. Wood RA, Segall N, Ahlstedt S, Williams PB. Accuracy of IgE antibody laboratory results. Ann Allergy Asthma Immunol 2007; 99:34-41.

36. Szecsi PB, Stender S. Comparison of immunoglobulin e measurements on IMMULITE and ImmunoCAP in samples consisting of allergen-specific mouse-human chimeric monoclonal antibodies towards allergen extracts and four recombinant allergens. Int Arch Allergy Immunol 2013;162: 131-4.

37. Graham F, Bégin P, Paradis L, Lacombe-Barrios J, Paradis J, Des Roches A. Comparison of immunoCAP and immulite serum specific IgE assays for the assessment of egg allergy. Allergy Asthma Clin Immunol 2016;12:29.

38. Hamilton RG. Clinical laboratory assessment of immediate-type hypersensitivity. J Allergy Clin Immunol 2010;125:S284-96.

39. Altmann F. Coping with cross-reactive carbohydrate determinants in allergy diagnosis. Allergo J Int 2016;25:98-105.

40. Ramón Lopez Salgueiro, Raquel Aparicio, Ana Giner Valero, Noelia Colomer, Ma Dolores Hernánez, F. de Rojas. CCD’s sensitization in a Mediterranean area. Allergy 2014 Sep;69(Suppl 99):1-619.

41. Holzweber F, Svehla E, Fellner W, Dalik T, Stubler S, Hemmer W, et al. Inhibition of IgE binding to cross-reactive carbohydrate determinants enhances diagnostic selectivity. Allergy Eur J Allergy Clin Immunol 2013;68:1269-77.

42. Nowakowska-Świrta E, Wiszniewska M, Walusiak-Skorupa J. Allergen-specific IgE to recombinant latex allergens in occupational allergy diagnostics. J Occup Health 2019;61: 378-86.

43. Grzywnowicz M, Majsiak E, Gaweł J, Miśkiewicz K, Doniec Z, Kurzawa R. Inhibition of cross-reactive carbohydrate determinants in allergy diagnostics. In Advances in experimental medicine and biology. New York LLC: Springer; 2018. 75-9 pp.

44. Sastre J. Molecular diagnosis in allergy. Clin Exp Allergy 2010;40: 1442-60.

45. Eller E, Bindslev-Jensen C. Clinical value of component-resolved diagnostics in peanut-allergic patients. Allergy Eur J Allergy Clin Immunol 2013;68:190-4.

46. Alessandri C, Ferrara R, Bernardi ML, Zennaro D, Tuppo L, Giangrieco I, et al. Diagnosing allergic sensitizations in the third millennium: why clinicians should know allergen molecule structures. Clin Transl Allergy 2017;7. https://doi.org/10.1186/ s13601-017-0158-7.
47. Vidal C, Enrique E, Gonzalo A, Moreno C, Tabar Al, Alcántara M, et al. Diagnosis and allergen immunotherapy treatment of polysensitised patients with respiratory allergy in Spain: an allergists' consensus. Clin Transl Allergy 2014;4. https://doi. org/10.1186/2045-7022-4-36.

48. Muñoz G, Subiza G-L. Diagnóstico, monitorización y tratamiento inmunológico de las enfermedades alérgicas [Internet]; 2020. [cited 2020 Apr 2], Available from: https://tienda.elsevier.es/ diagnostico-monitorizacion-y-tratamiento-inmunologico-de-lasenfermedades-alergicas-9788491132400.html.

49. Larsen JN, Broge L, Jacobi H. Allergy immunotherapy: the future of allergy treatment. Drug Discov Today 2016;21:26-37.

50. Sastre J, Landivar ME, Ruiz-García M, Andregnette-Rosigno MV, Mahillo I. How molecular diagnosis can change allergen-specific immunotherapy prescription in a complex pollen area. Allergy Eur J Allergy Clin Immunol 2012;67:709-11.

51. Tripodi S, Frediani T, Lucarelli S, MacR F, Pingitore G, Di Rienzo Businco A, et al. Molecular profiles of IgE to phleum pratense in children with grass pollen allergy: implications for specific immunotherapy. J Allergy Clin Immunol 2012;129. https://doi. org/10.1016/j.jaci.2011.10.045.

52. Mittermann I, Zidarn M, Silar M, Markovic-Housley Z, Aberer W, Korosec $\mathrm{P}$, et al. Recombinant allergen-based IgE testing to distinguish bee and wasp allergy. J Allergy Clin Immunol 2010; 125:1300-7.

53. Savi E, Peveri S, Cavaliere C, Masieri S, Montagni M. Laboratory tests for allergy diagnosis. J Biol Regul Homeost Agents 2018;32: 25-8.

54. Dodig S, Čepelak I. The potential of component-resolved diagnosis in laboratory diagnostics of allergy. Biochem Medica 2018;28:20501.

55. Tortajada-Girbés $M$, Mesa del Castillo $M$, Larramona H, Lucas JM, Álvaro Lozano M, Tabar Al, et al. Decision-making for pediatric allergy immunotherapy for aeroallergens: a narrative review. Eur J Pediatr. 2019. https://doi.org/10.1002/14651858.CD001936. pub2.

56. Barber D, De La Torre F, Feo F, Florido F, Guardia P, Moreno C, et al. Understanding patient sensitization profiles in complex pollen areas: a molecular epidemiological study. Allergy Eur J Allergy Clin Immunol 2008;63:1550-8.

57. Yu W, Freeland DMH, Nadeau KC. Food allergy: immune mechanisms, diagnosis and immunotherapy. Nat Rev Immunol 2016;16:751-65.

58. Rohr UP, Binder C, Dieterle T, Giusti F, Messina CGM, Toerien E, et al. The value of in vitro diagnostic testing in medical practice: a status report. PLoS One 2016;11. https://doi.org/10.1371/ journal.pone. 0149856 .

59. Bogavac-Stanojevic N, Jelic-Ivanovic Z. The cost-effective laboratory: implementation of economic evaluation of laboratory testing. J Med Biochem 2017;36:238-42.

60. Westwood M, Ramaekers B, Lang S, Armstrong N, Noake C, De Kock S, et al. Immunocap ${ }^{\circledR}$ ISAC and microtest for multiplex allergen testing in people with difficult to manage allergic disease: a systematic review and cost analysis. Health Technol Assess (Rockv) 2016;20. https://dx.doi.org/10.3310/hta20670.

61. Hermansson LL, Glaumann S, Borres M, Elenius M, Mascialino B, Hubben GA, et al. PMD33 cost-effectiveness of molecular IgE in vitro diagnostics (IVD) in children suspected with peanut allergy compared to double blind pacebo controlled food challenge (DBPCFC) in EU, USA and Japan. Value Health 2012;15:A68. 
62. Hermansson LL, Mascialino B, Glaumann S, Borres M, Hubben G, Nilsson $C$. Is molecular allergology cost-effective and cost saving in children with suspected peanut allergy compared to double blind placebo controlled food challenge (DBPCFC) and skin prick test in US, Europe and Asia? J Allergy Clin Immunol 2013;131: AB58.

63. Glaumann S, Hermansson LL, Mascialino B, Hubben G, Borres M, Nilsson C. Is molecular allergology cost-effective and cost saving in children with suspected peanut allergy compared to double blind placebo controlled food challenge (DBPCFC), open oral food challenge and skin prick test in Sweden? Clin Transl Allergy 2013; 3:1-2.

64. Hermansson LL, Mascialino B, Sastre J. PMD64 can molecular allergology improve allergen-specific immunotherapy adherence and patient quality of life in a complex pollen area? Value Heal 2012;15:A356.

65. Mascialino B, Hermansson LL, Sastre J. The role of molecular allergology in allergen-specific immunotherapy adherence and patient quality of life in a complex pollen area: a simulation model. J Allergy Clin Immunol 2013;131: AB201.

66. Reglamento UE. Del Parlamento Europeo y del Consejo sobre los productos sanitarios para diagnóstico in vitro y por el que se derogan la Directiva 98/79/CE y la Decisión 2010/227/UE de la
Comisión. R 2017/746 (5 abril 2017) [Internet], EU. Available from: https://www.boe.es/doue/2017/117/L00176-00332.pdf.

67. Marcellusi A, Viti R, Incorvaia C, Burastero SE, Mennini FS. Costi diretti e indiretti associati a malattie allergiche respiratorie in Italia. Uno studio probabilistico di cost of illness. Recenti Prog Med 2015;106:517-27.

68. Mayorga C, Ebo DG, Lang DM, Pichler WJ, Sabato V, Park MA, et al. Controversies in drug allergy: in vitro testing. J Allergy Clin Immunol 2019;143:56-65.

69. Bulat Lokas S, Plavec D, Rikić Pišković J, Živković J, Nogalo B, Turkalj M. Allergen-specific IgE measurement: intermethod comparison of two assay systems in diagnosing clinical allergy. J Clin Lab Anal [Internet] 2017;31:e22047.

70. Popescu FD, Vieru M. Precision medicine allergy immunoassay methods for assessing immunoglobulin E sensitization to aeroallergen molecules. World J Methodol [Internet] 2018;8:17-36.

Material Supplementario: La versión en línea de este artículo ofrece material suplementario (https://doi.org/10.1515/almed-2020-0022).

Nota del artículo: La versión traducida del artículo puede encontrarse aquí: https://doi.org/10.1515/almed-2020-0051 